\title{
A Review of Image Enhancement Techniques for Underwater Images
}

\author{
Khushboo Khurana ${ }^{1 *}$ and Neha Tirpude ${ }^{2}$ \\ ${ }^{1}$ Shri Ramdeobaba College of Engineering and Management, Nagpur \\ Indian Institute of Information Technology (IIIT), Nagpur, India
}

\section{ABSTRACT}

This paper presents a review of underwater image enhancement techniques which serves as the foundation of a variety of underwater imaging applications including the study of aquatic life forms, underwater object detection and classification or simply an improvement in the appearance of underwater images. Unlike regular imaging in a normal environment, underwater imaging suffers from reduced clarity, entailed by scattering and absorption of light. There are regions with non-uniform illumination and prominent bluish and greenish tones depending on the wavelength of light. The quality of underwater images also depends on the photographic device used to capture the image, turbidity of water. This paper discusses the different types of enhancement brought about by varied underwater image enhancement techniques.

\section{KEY WORDS: UNDERWATER IMAGE ENHANCEMENT, CONTRAST CORRECTION, COLOUR CORRECTION.}

\section{INTRODUCTION}

Underwater imaging is progressively becoming more significant due to the wide range of applications such as monitoring of underwater structures, to study various aquatic life forms like fishes, coral reefs, etc., to search for the abundant mineral and biological resources present in water bodies, aquatic robot inspection, underwater archaeology and so on. However, the images captured underwater are of degraded quality. The suspended particles in the water cause scattering of light. Also, the light is absorbed differently based on the frequency of light. Moreover, colour distortion, contrast degradation, and low visibility are prominent issues in the underwater images. Colour distortion is mostly caused by the way different light wavelengths travel in water. This makes

\section{ARTICLE INFORMATION}

Received 14th Oct 2020 Accepted after revision 27th Dec 2020 Print ISSN: 0974-6455 Online ISSN: 2321-4007 CODEN: BBRCBA

Thomson Reuters ISI Web of Science Clarivate Analytics USA and Crossref Indexed Journal

\section{Clarivate
Analytics}

NAAS Journal Score 2020 (4.31) SJIF: 2020 (7.728)

A Society of Science and Nature Publication,

Bhopal India 2020. All rights reserved.

Online Contents Available at: http//www.bbrc.in/

Doi: http://dx.doi.org/10.21786/bbrc/13.14/10 the colour of underwater images appear bluish-green. Whereas contrast is degraded as the light is randomly attenuated and scattered.

The nature of the underwater image can be corrected and made better for drawing out information for further analysis. The image quality can be enriched by the application of image restoration (Zhang, et. al., 2018) or image enhancement. Image restoration methods proceed by removing the noise and then apply the inverse degradation process to improve the underwater image quality. Whereas, image enhancement deals with the better appearance of the image from the human perspective. This paper presents a review of various underwater image enhancement methods.

Image enhancement techniques refine a given image such that the resulting image is easier to perceive for the human or more likely to be detected by automated image analysis systems. The key issues that need to be addressed by the image enhancement techniques for the underwater image are to improve visibility, enhance the image colour, contrast, to preserve the naturalness of the image, and enhance the object prominence. There are various image enhancing frameworks that are based on

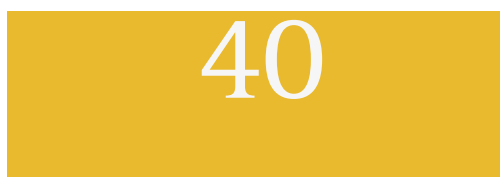


white balance, colour correction, histogram equalization, fusion-based methods, and Retinex-based Algorithms. In the literature, various underwater images are considered that have low visibility, low contrast, non-uniform illumination, blur, and light spot (Zheng L, et. al., 2016), images affected by blue-green light (Yu, Haifeng, et al., 2020), images having dark and light areas (Soni, Om Kumari, et al., 2020), images with haze (Ancuti, Codruta 0., et al., 2017) and acoustic images with non-uniform illumination (Sharumathi, K., and R. Priyadharsini, 2016), etc. Although a variety of images are used, most of the work that addresses the underwater image enhancement use random images from the internet to show the results for their proposed method. There are only a few available public datasets.

An open image dataset called TURBID has been created (Duarte, A., et al., 2016), to add value to research in underwater images. The dataset consists of three groups of images named as Milk (containing 20 images), DeepBlue (containing 20 images) and Chlorophyll (containing 42 images). Another dataset called UIEB dataset includes 890 underwater images that contain both the raw and its respective high-quality images and another set of 60 challenging underwater images (C. Li, et al., 2019). The image enhancement techniques are discussed in the next section followed by our conclusions in section 3 .

Literature Review: In literature, the problem of underwater image enhancement is addressed by utilizing either multiple images (Narasimhan SG, Nayar SK, 2003) or a single image. Various techniques take the advantage of the presence of multiple images and apply image fusion to get the enhanced image. One such target enhancement technique is presented in (Tian H, et al., 2018). An algorithm for contrast recovery is proposed (Narasimhan SG, Nayar SK, 2003) for weather degraded images, utilizing two images. Although the methods utilizing two images provide good results, they require multiple images captured from the same scene. Moreover, a point-wise fusion between different images is required, restricting the applicability of these methods to various real-life applications. The image enhancement techniques in which not more than one image is required for further processing are more popular since no supplementary images are needed to proceed. Thereby, we discuss only the single image-based enhancement techniques. A summary of the latest and significant methods is furnished in the table below.

Table 1 . Summary of Underwater image enhancement techniques

\begin{tabular}{|c|c|c|}
\hline Technique & $\begin{array}{l}\text { Enhancement } \\
\text { Handled }\end{array}$ & $\begin{array}{l}\text { Evaluation } \\
\text { Parameter }\end{array}$ \\
\hline $\begin{array}{l}\text { CLAHE and USM } \\
\text { (Zheng L, et al., 2016) }\end{array}$ & $\begin{array}{c}\text { Contrast and } \\
\text { overall intensity }\end{array}$ & Histogram \\
\hline $\begin{array}{l}\text { Underwater white balancing } \\
\text { and Multi-scale fusion } \\
\text { (Ancuti, Codruta O., et al., 2017) }\end{array}$ & $\begin{array}{l}\text { Image dehazing, } \\
\text { colour contrast and } \\
\text { edge sharpness }\end{array}$ & $\begin{array}{l}\text { Laplacian contrast, } \\
\text { weight Saliency weight, } \\
\text { Saturation weight }\end{array}$ \\
\hline $\begin{array}{l}\text { Contourlet transform } \\
\text { (Sharumathi, K., and R. } \\
\text { Priyadharsini, 2016) }\end{array}$ & $\begin{array}{c}\text { Removal of speckle } \\
\text { noise, contrast } \\
\text { enhancement for images } \\
\text { having dark shadows }\end{array}$ & $\begin{array}{l}\text { Point discontinuities, } \\
\text { line, edge, curve, contour }\end{array}$ \\
\hline $\begin{array}{l}\text { AHE with HE } \\
\text { (Duarte, A., et al., 2016) } \\
\text { CLAHE and percentile } \\
\text { methodologies } \\
\text { (Garg, et al., 2018) }\end{array}$ & $\begin{array}{l}\text { Local contrast } \\
\text { enhancement } \\
\text { Colour and } \\
\text { contrast }\end{array}$ & $\begin{array}{c}\text { Histogram and } \\
\text { pixel values } \\
\text { Root Mean Squared } \\
\text { Error (RMSE) and entropy }\end{array}$ \\
\hline $\begin{array}{l}\text { DCP with ACCLAHE and } \\
\text { HF (Dixit, et al., 2016) }\end{array}$ & $\begin{array}{l}\text { Contrast, edge } \\
\text { enhancement and blur }\end{array}$ & PSNR \\
\hline
\end{tabular}

Amongst underwater image enhancement techniques, the Histogram equalization (HE) method is most soughtafter owing to its results and easiness. This method generates an image that has a uniform distribution of probabilities of gray levels. HE helps in improving the image, also it doesn't require additional data regarding the origin of degradation and does not involve the constituents of the image. This technique yields better results in cases where the pixel intensities are spread over coequally across the image, but does not satisfactorily enhance the local details. The traditional HE method was enhanced by (Ketcham, David J., 1976) as the Adaptive Histogram Equalization (AHE). The AHE partitions out the image into blocks, such that every block is subject to computation of histogram equalization mapping. This computed mapping is delegated to its central pixel. In cases of images having areas of comparatively lower range of intensity, the algorithm performs well, it inflates the noise, in all other cases, it has better results.

Another method that works over the resultant two channels RGB and HSV model obtained after modification 
of histogram is presented in (Ghani, et al., 2015). The RGB contrast stretching is performed within the bounds of Rayleigh Distribution. The authors demonstrate that there has been an observed increase in the contrast of the tested data with lowered noise levels. This technique (Ghani, et al., 2015) was found to deliver an average mean square error (MSE) as 76.76 and peak signal to noise ratio (PSNR) as 31.13.

The Contrast-Limited Adaptive Histogram Equalization (CLAHE) (Reza A M., et al., 2004) approach reduces noise amplification. It also involves the partitioning of the grayscale image into blocks as in HE method. However, a contrast limiting technique and noise reduction is also applied. Moreover, the amplification in the noise signal is controlled by applying a threshold to the image histogram. Zheng et al. (Zheng L, et al., 2016) worked on a single underwater image by utilizing the CLAHE algorithm and unsharp mask (USM) transforms for image enhancement. The images obtained from these algorithms are fused by simple weighted blending. The enhanced images obtained from this technique are superior to the traditional histogram equalization and homomorphic filters methods.

Diksha Garg, et al. (Garg, et al., 2018) presented an image enhancement method that blends CLAHE and Percentile methodologies. More specifically, CLAHE with RGB and HSV channels are used with percentile technique. The images with degraded contrast and shading quality were enhanced, by correction in colour and contrast. Moreover, the results obtained are better than the Histogram Equalization, Dehazing, Percentile, CLAHE methods. In the survey of underwater image enhancement techniques (Soni, Om Kumari, et al., 2020), it was concluded that CLAHE was better in avoiding excess amplification of noise as compared to AHE alone. The study also inferred that Brightness Preserving Bi-Histogram Equalization (BBHE) was good at preserving the mean brightness alongside assisting in the enhancement of contrast.

Multi-scale fusion: Zhang et al. (Zhang, et al., 2017) have proposed an underwater image enhancement approach based on multi-scale fusion strategy by first applying image restoration and then enhancement. After obtaining the restored image on the base of the underwater image model, white balance is done to get the enhanced image of the restored image. Finally, these two are fused by application of a multi-scale fusion approach. Saturation and contrast metrics are used to weight each input while performing fusion. Also, this technique leads in reduced execution time.

In another technique by Ancuti et al., single images that are degraded due to underwater noise are used as input. Colour-compression and white-balance are applied to the original image to obtain two images. These images are fused, followed by the definition of their corresponding image weight maps so that the details of edges and contrast from the input images are derived in the resultant image (Sharumathi, K., and R. Priyadharsini, 2016). Only lower frequency constituents of the output image are used to restrict sharper weight map transformation followed by multi-scale fusion of images. The technique proposed is not dependent on the configuration of the camera that was used to capture the underwater images and gave better contrast Et sharpness.

Other Algorithms for Contrast enhancement and colour cast: Chiang et al. (Chiang, et al., 2017) concluded the effect of particles present in water that make the light rays scatter frequently when it travels underwater and that the colour tone of the water is also affected by varying wavelengths which affect the light absorption feature thus giving underwater images the typical shades of colour and contrast. Moreover, in the research work (Chiang, et al., 2017), underwater images have been assumed to contain the possibility of additional lighting sources. These images are then subject to the process of dehazing, after which the image-depth map is estimated and segmentation of image objects is done. The travelling light rays are prone to events of weakening of the wavelengths and hence are colour-corrected by adjustment of the additional light sources. The article also states that the water-depth levels can be estimated from the background light channels.

In another paper (Sharumathi, K., and R. Priyadharsini, 2016), acoustic images have been considered as candidates for underwater image enhancement. They have observed that acoustic images commonly suffer from noise which is in the form of speckled patterns caused due to the granularity of the sea-bed. The authors mention that the contourlet transform can be used to target discontinuities in points and linear structures by firstly applying the Laplacian pyramid followed by the usage of a directional filter bank. Most of the underwater image enhancement algorithms for image dehazing try to correct the global background light. The Dark Channel Priority (DCP) algorithm has been widely used as an image dehazing algorithm (He K, et al., 2011). However, the DCP algorithm is not sufficient to directly find the image depth. Consequently, the underwater image enhancement algorithms that obtain transmittance are proposed (Peng Y-T, et al., 2017). These methods obtain the depth of the image and depend on a single transmission map to obtain the depth information corresponding to the image, still, limiting the applicability to some specific underwater environment.

Another method for underwater image dehazing is presented in (Soni, Om Kumari, et al., 2020) that uses a double transmission map. It proceeds by performing homomorphic filtering followed by application of double transmission map and finally dual-image wavelet fusion is done. Homomorphic filtering is applied to remove the colour deviation. Depth map calculates the difference between the light and dark channels to produce an enhanced image. The resultant image obtained is combined with the image obtained by dark channel, by application of dual-image wavelet fusion technique. Moreover, CLAHE method is applied to obtain the resultant images with a better contrast. This method 
improves the effect on entropy, average gradient and the estimation of the colour image quality. The method surpasses the results obtained by DCP, histogram equalization (HE), and CLAHE algorithms.

A method of image enhancement using dark channel prior (DCP) with adaptively clipped contrast limited histogram equalization (ACCLAHE) and homomorphism filtering (HF) is presented in (Dixit, et al., 2016) for enhancing the image by correcting illumination that is not spread evenly, lowering noise and boosting of contrast. A foggy RGB image is considered as input and the blur region is estimated using DCP and removed. It is done by applying a maximum filter to determine the atmospheric light and to get the transmission map. Then this refined transmission map is used for smoothing the image. After that ACCLAHE is applied to perform image enhancement. From the local histogram of the sub-image, peak bin height is considered and the clipped pixels are spread uniformly to each gray-level. Finally, HF is used to derive better edges.

In (Lu, et al., 2015), the technique proposed focuses on underwater images that have been captured in turbid water. The deviation in attenuation in the route of propagation is balanced out. The technique applies an alternative form of the DCP for estimation of transmission, an ambient light estimator that works on colour lines and a locally adaptive filtering algorithm for enhancing shallow water images. The distortion in colour of underwater images is rectified using a colour correction algorithm targeting the spectrum-based properties to retrieve the colour distortion. The output images resulted in enhanced edges with an enhancement in overall contrast. Underwater images suffer from the presence of noise commonly due to the clarity of water and photography standards. Also, underwater images result in substandard quality because of inferior contrast and absence of well-distributed lighting. P. Sahu et al. (Hung-Yu, et al., 2005) have devised a methodology based on median filtering through which there was an observed reduction in noise grains present in the underwater images. This improvement in the image quality also assisted in decently projecting the waterdepth map through the use of forward unsharp masking, early selection of a dark channel and use of colour correction method.

In the underwater ambience, there is a prevalence of colour cast issue that is the dominance of blue/ green colour, and a colour equalization method has been proposed to address this problem (Iqbal K, et al., 2010). A scale factor based on the dominating colour plane in the RGB colour space is used to even up the remnant colours. However, this blind colour equalization deteriorates the image colour quality. Beer's law is one of the methods that aims at lowering colour cast. It works by rectifying the pixel intensity through computation of the amount of light absorbed in water. The assumption made is that underwater objects are situated at the same depth level, thus helping computation of missing wavelengths. Although it is not a common situation in many cases that the objects are at the same depth or the medium is homogeneous, the method shows better image enhancement (Narasimhan SG, Nayar SK, 2003).

Retinex-based Algorithms: A Retinex-based variational framework is designed to enhance a single underwater image. A statistical colour correction method is proposed to cater to the problem of colour distortion. This is followed by application of a variational Retinex model to the obtained colour corrected image. Thereby the luminance is decomposed to obtain its constituentsreflectance and illumination. Finally, fuzz and underexposure are reduced by enhancing the reflectance and the illumination using the histogram method. To address colour shift and contrast degradation, a two-step algorithm is presented in (Fu X, et al. 2017). A colour correcting method that depends on a piecewise linear transformation is introduced to correct the colour. This is followed by application of a contrast improvement strategy that improves the contrast.

\section{CONCLUSION}

Thus from the underwater scenarios discussed in this review, it can be concluded that the necessity of enhancing the underwater images arises from the degradation in the quality of those images due to issues in contrast, light-absorption \& attenuation, clarity/turbidity of the water, noise present in captured images and also the distance at which the camera has been placed. Further enhancement may be required to target and detect potential objects underwater. It can also be concluded that wavelet and curvelet transformation methods are found to perform better in removing noise from acoustic images. In cases where the fusion principle was used, the image resulting enhancement was found to be useful for tough underwater applications of computer vision. The underwater dehazing and contrast enhancement can be handled better by use of HF, CLAHE along with DCP and transmission maps. Although a remarkable amount of progress has been made in the field of underwater research and investigation, there is a huge scope for the development of image and video processing techniques that can assist this underwater exploration. Moreover, there is a need to build the underwater image datasets, so that the assessment can be carried out on the same set of images.

\section{REFERENCES}

Ancuti, Codruta 0., et al. 2017. Colour balance and fusion for underwater image enhancement. IEEE Transactions on image processing 27.1: 379-393.

C. Li, C. Guo, W. Ren, R. Cong, J. Hou, S. Kwong, D. Tao. 2019. An Underwater Image Enhancement Benchmark Dataset and Beyond. IEEE Trans. Image Process. 29:4376-4389

Chiang, John Y., and Ying-Ching Chen. 2011. Underwater image enhancement by wavelength compensation and dehazing. IEEE Transactions on Image Processing 21.4: 1756-1769. 
Dixit, Sonal, Sandeep Kumar Tiwari, and Pankaj Sharma. 2016. Underwater image enhancement using DCP with ACCLAHE and homomorphism filtering. 2016 International Conference on Signal Processing, Communication, Power and Embedded System (SCOPES). IEEE.

Duarte, A., Codevilla, F., Gaya, J.D.O. and Botelho, S.S., 2016, April. A dataset to evaluate underwater image restoration methods. In OCEANS 2016-Shanghai (pp. 1-6). IEEE.

Garg, Diksha, Naresh Kumar Garg, and Munish Kumar. 2018. Underwater image enhancement using blending of CLAHE and percentile methodologies. Multimedia Tools and Applications 77.20: 26545-26561.

Fu X, Fan Z, Ling M, Huang Y, Ding X. 2017. Two-step approach for single underwater image enhancement. In Proc. International Symposium on Intelligent Signal Processing and Communication Systems, pp. 789-794.14

Ghani, Ahmad Shahrizan Abdul, and Nor Ashidi Mat Isa. 2015. Underwater image quality enhancement through integrated colour model with Rayleigh distribution. Applied soft computing 27: 219-230.

He K, Sun J, Tang X. 2011. Single image haze removal using dark channel prior. IEEE Trans Pattern Anal Mach Intell 33(12):2341-2353

Hung-Yu, Pie-Yin Chen, Chien-Chuan Huang and YaZhu Zhuang. 2005. Second International Conference on Innovations in Bio computing and Applications.

Iqbal K, Odetayo M, James A, Salam R A and Talib A. 2010. Enhancing the low quality images using unsupervised colour correction method. IEEE Int. Conf. Syst. Man Cybern. (SMC), pp 1703-1709

Ketcham, David J. Real-time image enhancement techniques. 1976. Image processing. International Society for Optics and Photonics.

Lu, Huimin, et al. 2015. Contrast enhancement for images in turbid water. JOSA A 32.5: 886-893.
Narasimhan SG, Nayar SK. 2003. Contrast restoration of weather degraded images. IEEE Trans Pattern Anal Mach Intell 25(6):713-724.

Peng Y-T, Cosman PC. 2017. Underwater image restoration based on image blurriness and light absorption. IEEE Trans Image Process 26(4):15791594

Reza A M. 2004. Realization of the contrast limited adaptive histogram equalization (CLAHE) for realtime image enhancement [J]. Journal of VLSI signal processing systems for signal, image and video technology. 38(1): 35-44.

Sharumathi, K., and R. Priyadharsini. 2016. A survey on various image enhancement techniques for underwater acoustic images.” 2016 International Conference on Electrical, Electronics, and Optimization Techniques (ICEEOT). IEEE.

Soni, Om Kumari, and Jamvant Singh Kumare. 2020. A Survey on Underwater Images Enhancement Techniques. IEEE 9th International Conference on Communication Systems and Network Technologies (CSNT). IEEE.

Tian H, Zhu J, Tan S, Zhang Y, Zhang Y, Li Y, Hou X. 2018. Rapid underwater target enhancement method based on polarimetric imaging. Opt Laser Technol 108:515-520

Yu, Haifeng, et al. 2020. Underwater image enhancement based on DCP and depth transmission map. Multimedia Tools and Applications. 1-18.

Zhang, Mohua, and Jianhua Peng. 2018. Underwater image restoration based on a new underwater image formation model. IEEE Access 6: 58634-58644.

Zhang, Can, Xu Zhang, and Dawei Tu. (2017) Underwater image enhancement by fusion." International Workshop of Advanced Manufacturing and Automation. Springer, Singapore.

Zheng L, Shi H, Sun S. 2016. Underwater image enhancement algorithm based on CLAHE and USM. Proc IEEE Int Conf Inform Automation (ICIA), 585-590 